\title{
Rapid and early $\alpha$-fetoprotein and des- $\gamma$-carboxy prothrombin responses to initial arterial infusion chemotherapy predict treatment outcomes of advanced hepatocellular carcinoma
}

\author{
KENJI OYAMA ${ }^{1}$, MASAHIKO KODA ${ }^{2}$, TAKAAKI SUGIHARA ${ }^{2}$, MANABU KISHINA ${ }^{2}$, KENICHI MIYOSHI ${ }^{2}$, \\ TOSHIAKI OKAMOTO ${ }^{2}$, MASANORI HODOTSUKA ${ }^{2}$, YUKI FUJISE ${ }^{2}$, TOMOMITSU MATONO ${ }^{2}$, \\ SHIHO TOKUNAGA ${ }^{2}$, KINYA OKAMOTO ${ }^{2}$, KEIKO HOSHO ${ }^{2}$, JUNICHI OKANO ${ }^{2}$ and YOSHIKAZU MURAWAKI ${ }^{2}$ \\ ${ }^{1}$ Tottori University Hospital Cancer Center; ${ }^{2}$ Division of Medicine and Clinical Science, Department of Multidisciplinary \\ Internal Medicine, Faculty of Medicine, Tottori University, Yonago, Tottori 683-8504, Japan
}

Received December 8, 2014; Accepted February 26, 2015

DOI: $10.3892 / \mathrm{mco} .2015 .523$

\begin{abstract}
The aim of the present study was to predict the effects of transarterial infusion (TAI) chemotherapy based on early changes in $\alpha$-fetoprotein (AFP) and des- $\gamma$-carboxy prothrombin (DCP) in patients with advanced hepatocellular carcinoma (HCC). Seventy-four patients who underwent TAI with cisplatin, 5-fluorouracil, mitomycin $\mathrm{C}$ and epirubicin for advanced $\mathrm{HCC}$ were enrolled. Antitumor responses were evaluated 6 months after TAI. Rapid and early responses were defined as the ratio of AFP or DCP after 1 week and 1 month compared to baseline. A total of 5, 10, 17 and 42 patients had complete response (CR), partial response (PR), stable disease (SD) or progressive disease (PD), respectively. Early AFP response was significantly lower in the $\mathrm{CR}+\mathrm{PR}$ compared to the $\mathrm{SD}+\mathrm{PD}$ groups $(\mathrm{P}<0.01)$. The early DCP response was significantly lower in the $\mathrm{CR}+\mathrm{PR}$ compared to the $\mathrm{SD}+\mathrm{PD}$. The sensitivity and specificity of rapid and early AFP responses in the CR+PR were 0.78 and 0.72 , and 0.80 and 0.73 , respectively, and those of rapid and early DCP responses were 0.67 and 0.65 , and 0.77 and 0.71 , respectively. The combination of AFP and DCP responses had higher specificity compared to AFP or DCP alone responses. Patients were divided into responder and non-responder groups to evaluate the prediction of survival outcome. Early responders of AFP, DCP and AFP+DCP, who were divided based on the cut-off values of $\mathrm{CR}+\mathrm{PR}$ survived significantly longer than the non-responders $(\mathrm{P}<0.05)$. In conclusion, rapid or early responses of AFP and/or DCP levels 1 and 4 weeks after TAI chemotherapy helped to predict the treatment effects.
\end{abstract}

Correspondence to: Dr Masahiko Koda, Division of Medicine and Clinical Science, Department of Multidisciplinary Internal Medicine, Faculty of Medicine, Tottori University, 36-1 Nishi-machi, Yonago, Tottori 683-8504, Japan

E-mail:masakoda@med.tottori-u.ac.jp

Key words: hepatocellular carcinoma, transarterial infusion chemotherapy, $\alpha$-fetoprotein, des- $\gamma$-carboxy prothrombin, treatment response

\section{Introduction}

Hepatocellular carcinoma (HCC) is the fifth and seventh most common cancer in men and women, respectively (1) and the third leading cause of cancer mortality worldwide (2). Although radiofrequency ablation (RFA), percutaneous ethanol injection (PEI), surgical resection and liver transplantation can be curative, $\sim 80 \%$ of patients with HCC are not candidates for these strategies due to having advanced or metastatic disease at the time of presentation (3). Multiple advanced HCC has been treated using transcatheter arterial chemoembolization (TACE), but only in patients without portal vein tumor thrombus.

Sorafenib is a small molecular inhibitor of several tyrosine proteins and Raf kinases and is widely used to treat patients with advanced HCC with or without metastasis $(4,5)$. Sorafenib is now recommended as it confers survival benefits beyond the best supportive care (6), but only patients with Child-Pugh A grade are candidates (7) due to the adverse events such as liver damage (8). Additionally, it is not widely administered as it is extremely expensive (9).

Patients with advanced HCC are often treated with transarterial infusion (TAI) chemotherapy according to indications. However, TAI has not yet been established as a standard treatment for advanced HCC without extrahepatic metastasis, as its effects have not yet been supported by concrete evidence generated from randomized controlled trials. Previous studies have identified complete (CR) and partial (PR) response rates after TAI of 27 to $40 \%$, respectively $(10,11)$ and a clearly improved prognosis for patients with stable (SD) or progressive disease (PD). However, repeated TAI for patients with SD or PD imposes physical, psychological and economic burdens. Therefore, patients with CR or PR should be differentiated from those with SD or PD during the earliest phase of the treatment.

$\alpha$-Fetoprotein (AFP) and des- $\gamma$-carboxy prothrombin (DCP) are established tumor markers for HCC and they are useful for diagnosis $(12,13)$. However, whether or not changes in their levels can serve as markers of responses to treatment remains controversial. Several recent studies have identified that changes in AFP levels following systemic chemotherapy, chemoembolization and radioembolization, may predict the 
effects of treatment (14-16). Numerous studies have proven that AFP and DCP are useful not only as tumor markers, but also as prognostic factors for $\operatorname{HCC}(17,18)$.

Changes in tumor markers following anticancer therapies closely correlate with treatment effects in various types of solid tumors. Predicting treatment effects early will aid physicians in determining whether current therapy should be continued or changed.

However, correlations between AFP and DCP responses and survival outcomes in patients treated with TAI have not yet been established.

Therefore, the present study aimed to determine whether or not AFP and DCP responses during the early phase of treatment can predict the prognosis of patients with advanced HCC. Correlations were evaluated between AFP and DCP responses to treatment effects and survival rates in patients with $\mathrm{HCC}$ treated with TAI.

\section{Materials and methods}

Patients. A total of 74 patients with HCC confirmed by transcatheter arterial angiography and who received TAI comprising $25 \mathrm{mg}$ cisplatin, $500 \mathrm{mg} 5$-fluorouracil, $6 \mathrm{mg}$ mitomycin $\mathrm{C}$ and $30 \mathrm{mg}$ epirubicin were enrolled at the Tottori University Hospital (Tottori, Japan) between January 2004 and December 2012. None of the patients were candidates for liver transplantation, surgical resection, PEI, RFA or TACE as multiple tumors were involved with one or both of the hepatic lobes with or without portal vein tumor thrombosis. Patients were treated with TAI once each month after initial infusion chemotherapy. Twenty-one patients received other treatments with subsequent TAI, and sorafenib was administered to two patients after repeated TAI. Fifteen patients succumbed within 6 months after initial TAI due to disease progression, and they were divided into PD. The study was approved by the Ethics Committee of the Tottori University Faculty of Medicine (no. 1863).

Protocols. Serum AFP and DCP levels were measured before the initial TAI at baseline and at 1 and 4 weeks after TAI. A rapid response rate was defined as the ratio of AFP or DCP value at 1 week after initial TAI compared to the baseline values and the early response rate was the ratio at 4 weeks. Serum AFP and DCP levels were measured using the Access AFP micro-particle enzyme immunoassay (Beckman Coulter, Brea, CA, USA) and Lumipulse presto PIVKA-II (Eidia Co., Ltd., Tokyo, Japan), respectively. Patients with normal baseline AFP $(<10 \mathrm{ng} / \mathrm{ml})$ and DCP $(<40 \mathrm{mAU} / \mathrm{ml})$ levels were excluded from the response evaluation. The effects of treatment and disease progression were assessed using contrast-enhanced computed tomography or magnetic resonance imaging every 8-12 weeks.

Response evaluation criteria. Antitumor responses were evaluated according to the standards of the Liver Cancer Study Group of Japan (19) at 6 months after the initial treatment. Briefly, a CR was defined as the total radiological absence of all the known lesions. A PR was defined as a decrease of $\geq 50 \%$ in the product of two perpendicular diameters of the largest tumor nodule for $>4$ weeks without the appearance of new lesions, or progression of existing lesions. A SD was defined as $<50 \%$ decrease or not $>25 \%$ increase in the product of two perpendicular diameters of the largest tumor nodule. A PD was defined as an increase of $>25 \%$ in the product of two perpendicular diameters of the largest tumor nodule or one of the measurable lesions, or the appearance of new lesions. Patients who succumbed due to HCC progression before 6 months were categorized as having PD.

Statistical analysis. Data were statistically analyzed using Stat Flex version 6 (Artech, Osaka, Japan). A two-sided $\mathrm{P}<0.05$ was considered to indicate a statistically significant difference. The amount of change in AFP and DCP between the CR and $\mathrm{PR}+\mathrm{SD}+\mathrm{PD}$, and between the $\mathrm{CR}+\mathrm{PR}$ and $\mathrm{SD}+\mathrm{PD}$ groups was compared using the Mann-Whitney U test and categorical variables were compared using the Kruskal-Wallis test. Overall survival was calculated using the Kaplan-Meier method and compared using the log-rank test. Sensitivity, specificity, positive predictive values (PPV) and negative predictive values were determined from receiver operating characteristic (ROC) curves.

\section{Results}

Antitumor responses at 6 months after TAI. Table I shows the characteristics of the patients. Among the enrolled patients, 5 (6.8\%), 10 (13.5\%), 17 (23.0\%) and 42 (56.8\%) achieved CR, $\mathrm{PR}, \mathrm{SD}$ and $\mathrm{PD}$, respectively. The objective response rate of overall (CR+PR) was $20.3 \%$.

Survival outcomes according to treatment effects. Fig. 1 shows that the median overall survival of each group was $1,069,1,201$, 773 and 245 days for those who achieved CR, PR, SD and PD, respectively. Overall survival was longer for the CR compared to the $\mathrm{PR}+\mathrm{SD}+\mathrm{PD}$ group $(\mathrm{P}=0.054)$ and significantly longer for the $\mathrm{CR}+\mathrm{PR}$, compared to the $\mathrm{SD}+\mathrm{PD}$ group $(\mathrm{P}=0.0005)$.

Comparison of clinical characteristics according to treatment effects. Table II shows the clinical characteristics of the patients in each group. Age, gender and rate of liver cirrhosis prior to the initial TAI did not significantly differ among the four groups at baseline. Median baseline levels of AFP and DCP did not significantly differ among these groups $(\mathrm{P}=0.07$ and 0.86 , respectively). In total, 8, 7, 7, 5, 3, 2 and 2 patients each were treated with PEI, low-dose FP, radiation therapy, TACE, TS-1 (tegafur-gimeracil-oteracil potassium), sorafenib and RFA, respectively. Twenty-one patients were treated using these approaches following repeated TAI and 11 were treated only with these approaches.

Rapid and early response indices. Fig. 2 shows the rapid and early response indices of AFP and DCP following initial TAI. The rapid AFP indices of median were $0.69,0.83,1.01$ and 1.15 in CR, PR, SD and PD, respectively, and the early AFP response indices were $0.20,0.51,0.88$ and 1.43 in CR, PR, SD and PD, respectively. The rapid AFP response index was lower in the CR compared to the $\mathrm{PR}+\mathrm{SD}+\mathrm{PD}$ group $(\mathrm{P}=0.0717)$ and in the $\mathrm{CR}+\mathrm{PR}$ compared to the $\mathrm{SD}+\mathrm{PD}$ group $(\mathrm{P}=0.0690)$. The early AFP response index was lower in the $\mathrm{CR}$ compared to the $\mathrm{PR}+\mathrm{SD}+\mathrm{PD}$ group $(\mathrm{P}=0.0099)$ and in the $\mathrm{CR}+\mathrm{PR}$ compared to the $\mathrm{SD}+\mathrm{PD}$ group $(\mathrm{P}=0.0003)$. 
Table I. Patient characteristics.

\begin{tabular}{lc}
\hline Characteristics & Values \\
\hline Age, median years (range) & $67.7(38-89)$ \\
Gender, $\mathrm{n}$ (male/female) & $62 / 12$ \\
Etiology, $\mathrm{n}$ & \\
HBV & 23 \\
HCV & 34 \\
Alcohol & 10 \\
Others & 7 \\
Underlying liver disease, $\mathrm{n}$ & \\
Chronic hepatitis & 26 \\
Liver cirrhosis & 48 \\
Child-Pugh grade, $\mathrm{n}(\mathrm{A} / \mathrm{B} / \mathrm{C})$ & $31 / 15 / 2$ \\
HCC clinical stage, $\mathrm{n}(\mathrm{II} / \mathrm{III} / \mathrm{IVa} / \mathrm{IVb})$ & $15 / 26 / 25 / 8$ \\
Portal vein tumor thrombus, $\mathrm{n}(\%)$ & $20(27.0)$ \\
Hepatic vein tumor thrombus, $\mathrm{n}$ & 1 \\
Lymph node metastasis, $\mathrm{n}$ & 6 \\
Distant metastasis, $\mathrm{n}$ & \\
Lung & 5 \\
Bone & 3 \\
Adrenal gland &
\end{tabular}

Serum markers at baseline, median (range)

$$
\text { AFP, ng/ml }
$$$$
\mathrm{DCP}, \mathrm{mAU} / \mathrm{ml}
$$

Treatment following initial TAI, $\mathrm{n}$

$\begin{array}{ll}\text { TAI } & 29 \\ \text { TAI + others } & 21 \\ \text { Others } & 11 \\ \text { None } & 13\end{array}$

Treatment response, $\mathrm{n}(\%)$

CR

PR

SD

Survival outcome, $\mathrm{n}$

$\begin{array}{lr}\text { Succumbed } & 54 \\ \text { Alive } & 13 \\ \text { Unknown } & 7\end{array}$

$\mathrm{HBV}$, hepatitis B virus; $\mathrm{HCV}$, hepatitis C virus; $\mathrm{HCC}$, hepatocellular carcinoma; AFP, $\alpha$-fetoprotein; DCP, des- $\gamma$-carboxy prothrombin; TAI, transarterial infusion; PR, partial response; CR, complete response; $\mathrm{SD}$, stable disease; $\mathrm{PD}$, progressive disease.

The rapid DCP response indices of median were 0.49 , 1.22, 0.89 and 0.96 in CR, PR, SD and PD, respectively. The early DCP response indices were $0.12,0.51,0.63$ and 1.14 in $\mathrm{CR}, \mathrm{PR}, \mathrm{SD}$ and $\mathrm{PD}$, respectively. The rapid DCP response index was significantly lower in the $\mathrm{CR}$ compared to the $\mathrm{PR}+\mathrm{SD}+\mathrm{PD}$ group $(\mathrm{P}=0.0064)$. The early $\mathrm{DCP}$ response index was significantly lower in the $\mathrm{CR}$, compared to the
A

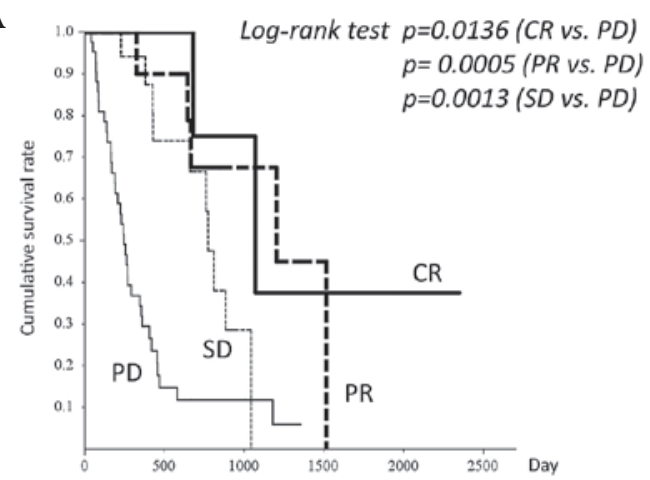

B

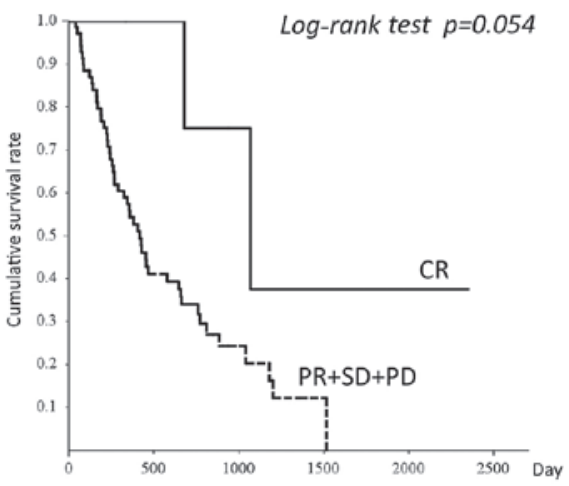

C

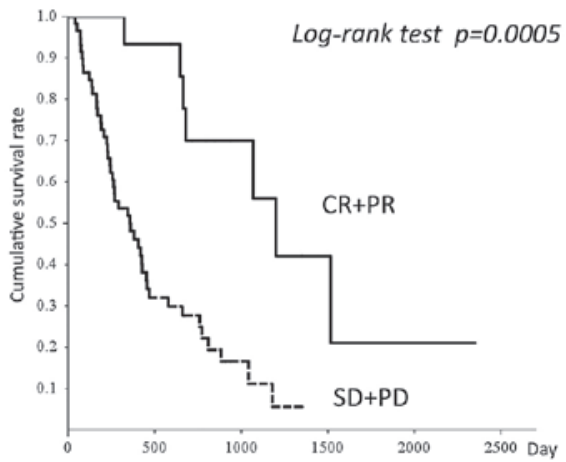

Figure 1. Kaplan-Meier analyses of overall survival according to objective responses. Overall survival was significantly shorter in (A) PD compared to the other groups $(\mathrm{P}<0.05)$ and longer for $(\mathrm{B}) \mathrm{CR}$ compared to $\mathrm{PR}+\mathrm{SD}+\mathrm{PD}$ $(\mathrm{P}=0.054)$ and for $(\mathrm{C}) \mathrm{CR}+\mathrm{PR}$ compared to $\mathrm{SD}+\mathrm{PD}(\mathrm{P}=0.0005)$. $\mathrm{PR}$, partial response; $\mathrm{CR}$, complete response; $\mathrm{SD}$, stable disease; $\mathrm{PD}$, progressive disease.

$\mathrm{PR}+\mathrm{SD}+\mathrm{PD}$ group and in the $\mathrm{CR}+\mathrm{PR}$, compared to the $\mathrm{SD}+\mathrm{PD}$ group $(\mathrm{P}<0.01)$.

Predicting performance based on the rapid and early response indices of $A F P$ and DCP. Fig. 3 shows the ROC curves for treatment responses ( $\mathrm{CR}$ vs. $\mathrm{PR}+\mathrm{SD}+\mathrm{PD}$ and $\mathrm{CR}+\mathrm{PR}$ vs. $\mathrm{SD}+\mathrm{PD})$. The areas under the ROC curves of early AFP or DCP responses were larger for the CR than for the $\mathrm{CR}+\mathrm{PR}$ group. The sensitivity and specificity for the CR group were 0.67 and 0.85 for a rapid AFP response (cut-off, 0.72), 1.00 and 0.89 for an early AFP response (cut-off, 0.46), 0.80 and 0.81 for a rapid DCP response (cut-off, 0.55 ) and 0.80 and 0.81 for an early DCP response (cut-off, 0.45 ), respectively. The sensitivity and specificity for the $\mathrm{CR}+\mathrm{PR}$ groups were 0.78 and 0.72 for a rapid AFP response (cut-off, 0.92), 0.80 and 0.73 for an early AFP response (cut-off, 1.02), 0.67 and 0.65 for a rapid DCP response (cut-off, 0.77) and 0.77 and 0.71 for an early DCP response (cut-off, 0.61), respectively (Table III). 
Table II. Patient characteristics according to treatment efficacy.

\begin{tabular}{lccccc}
\hline Characteristics & $\mathrm{CR}(\mathrm{n}=5)$ & $\mathrm{PR}(\mathrm{n}=10)$ & $\mathrm{SD}(\mathrm{n}=17)$ & $\mathrm{PD}(\mathrm{n}=42)$ & P-value \\
\hline Age, years & $66.8 \pm 11.5$ & $66.8 \pm 11.4$ & $70.0 \pm 9.1$ & $67.1 \pm 12.2$ & 0.86 \\
Gender, $\mathrm{n}$ (male/female) & $5 / 0$ & $9 / 1$ & $15 / 2$ & $33 / 9$ & 0.51 \\
$\begin{array}{l}\text { Etiology of liver disease, } \mathrm{n} \\
\text { alcohol/B/C/others }\end{array}$ & $0 / 3 / 2 / 0$ & $1 / 5 / 3 / 1$ & $5 / 3 / 7 / 2$ & $4 / 12 / 22 / 4$ & \\
Liver cirrhosis, $\mathrm{n}$ (no/yes) & $1 / 4$ & $4 / 6$ & $7 / 10$ & $14 / 28$ & 0.82 \\
Clinical stage, $\mathrm{n}$ (II/III/IVa/IVb) & $1 / 2 / 2 / 0$ & $3 / 3 / 4 / 0$ & $5 / 7 / 4 / 1$ & $6 / 14 / 15 / 7$ & \\
Baseline tumor markers, median (range) & & & & & \\
AFP, ng/ml & $395(46-70,254)$ & $49(13-35,730)$ & $20(11-53,110)$ & $270(11-162,579)$ & 0.07 \\
DCP, mAU/ml & $360(102-18,753)$ & $2,010(46-9,342)$ & $425(53-32,652)$ & $598(46-652,830)$ & 0.86 \\
Following treatment, $\mathrm{n}$ & & & & & \\
$\quad$ TAI/TAI + others/others/none & $2 / 3 / 0 / 0$ & $4 / 4 / 2 / 0$ & $11 / 5 / 1 / 0$ & $12 / 9 / 8 / 13$ & \\
\hline
\end{tabular}

TAI, transarterial infusion; PR, partial response; CR, complete response; SD, stable disease; PD, progressive disease; AFP, $\alpha$-fetoprotein; DCP, des- $\gamma$-carboxy prothrombin.
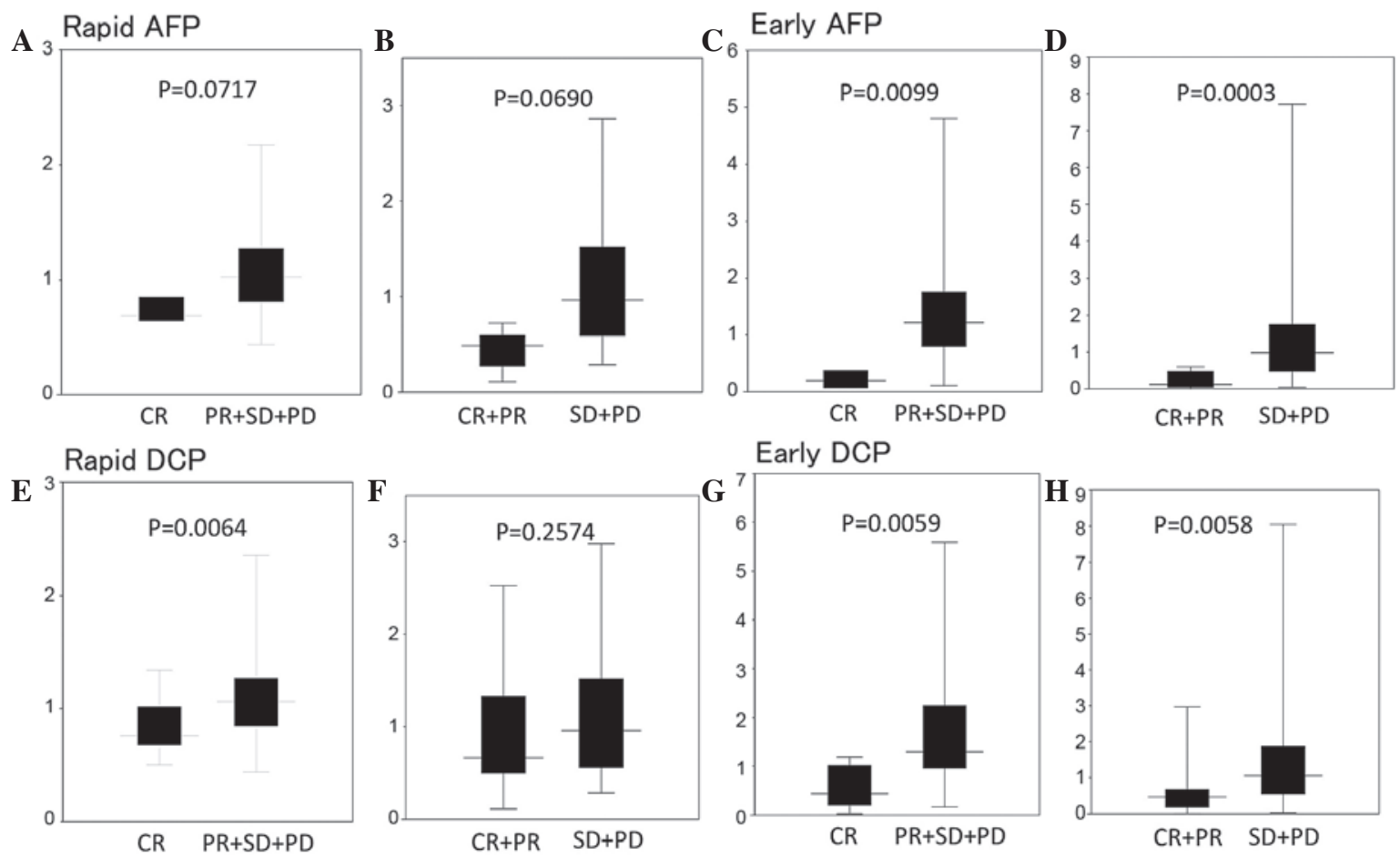

Figure 2. Rapid and early response indices of AFP and DCP following initial TAI. (A-D) Rapid and early AFP responses were lower in CR or CR+PR compared to $\mathrm{PR}+\mathrm{SD}+\mathrm{PD}$ or $\mathrm{SD}+\mathrm{PD}$. (E and F) Rapid DCP response was significantly lower in CR compared to PR+SD+PD. (G and H) Early DCP responses were significantly lower in $\mathrm{CR}$ or $\mathrm{CR}+\mathrm{PR}$ compared to $\mathrm{PR}+\mathrm{SD}+\mathrm{PD}$ or $\mathrm{SD}+\mathrm{PD}$. AFP, $\alpha$-fetoprotein; DCP, des- $\gamma$-carboxy prothrombin; TAI, transarterial infusion; $\mathrm{PR}$, partial response; $\mathrm{CR}$, complete response; $\mathrm{SD}$, stable disease; $\mathrm{PD}$, progressive disease.

Predicting performance based on a combination of AFP and $D C P$. Combining the AFP and DCP responses markedly increased the ability to predict $\mathrm{CR}$ and $\mathrm{CR}+\mathrm{PR}$ compared with each marker alone (Table IV). The cut-off values of AFP and DCP were the same as those used for the single marker analysis. The sensitivity and specificity were 0.67 and 0.98 for a rapid response, 1.00 and 1.00 for an early response for the CR group, 0.63 and 0.88 for a rapid response, and 0.75 and 0.88 for an early response, respectively, for the $\mathrm{CR}+\mathrm{PR}$ group. The
PPVs of rapid and early responses for CR were 0.67 and 1.00 , and those for $\mathrm{CR}+\mathrm{PR}$ were 0.50 and 0.55 , respectively.

Antitumor responses and survival outcomes according to subsequent treatments following initial TAI. The objective response rate of repeated TAI, repeated TAI + others and others as subsequent treatments were $20.7,33.3$ and $18.2 \%$, respectively. The median overall survival of each group was 659,646 , 418 and 140 days for those who received repeated TAI, repeated 
Table III. Predicting performance by AFP or DCP.

\begin{tabular}{|c|c|c|c|c|c|c|c|}
\hline Characteristics & Cut-off value & Sensitivity & Specificity & PPV & NPV & Hazard ratio & AUROC \\
\hline \multicolumn{8}{|l|}{ AFP } \\
\hline \multicolumn{8}{|l|}{ Rapid } \\
\hline $\mathrm{CR}$ & 0.72 & 0.67 & 0.85 & 0.20 & 0.98 & 11.3 & 0.81 \\
\hline $\mathrm{CR}+\mathrm{PR}$ & 0.92 & 0.78 & 0.72 & 0.35 & 0.94 & 9.2 & 0.69 \\
\hline \multicolumn{8}{|l|}{ Early } \\
\hline $\mathrm{CR}$ & 0.46 & 1.00 & 0.89 & 0.33 & 1.00 & NA & 0.95 \\
\hline $\mathrm{CR}+\mathrm{PR}$ & 1.02 & 0.80 & 0.73 & 0.38 & 0.95 & 10.8 & 0.87 \\
\hline \multicolumn{8}{|l|}{ DCP } \\
\hline \multicolumn{8}{|l|}{ Rapid } \\
\hline $\mathrm{CR}$ & 0.55 & 0.80 & 0.81 & 0.29 & 0.98 & 17.2 & 0.87 \\
\hline $\mathrm{CR}+\mathrm{PR}$ & 0.77 & 0.67 & 0.65 & 0.33 & 0.88 & 3.8 & 0.61 \\
\hline \multicolumn{8}{|l|}{ Early } \\
\hline CR & 0.45 & 0.80 & 0.81 & 0.27 & 0.98 & 16.7 & 0.87 \\
\hline $\mathrm{CR}+\mathrm{PR}$ & 0.61 & 0.77 & 0.71 & 0.42 & 0.92 & 8.3 & 0.75 \\
\hline
\end{tabular}

PPV, positive predictive value; NPV, negative predictive value; AUROC, area under receiver operating curve; PR, partial response; CR, complete response; NA, not applicable.

A

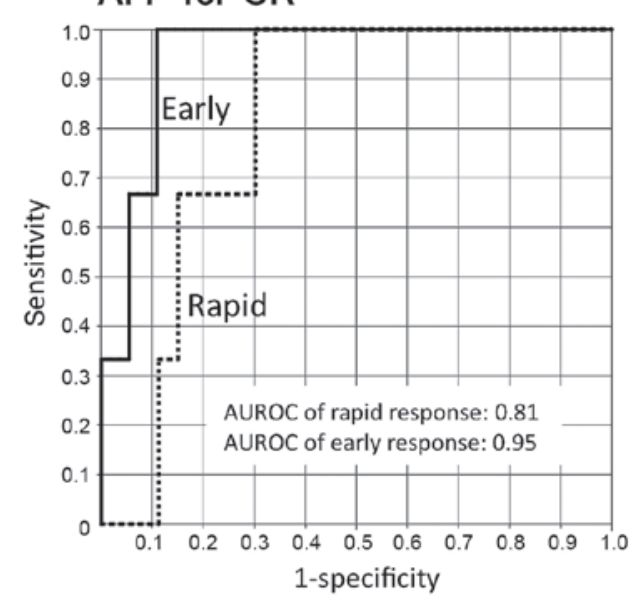

C

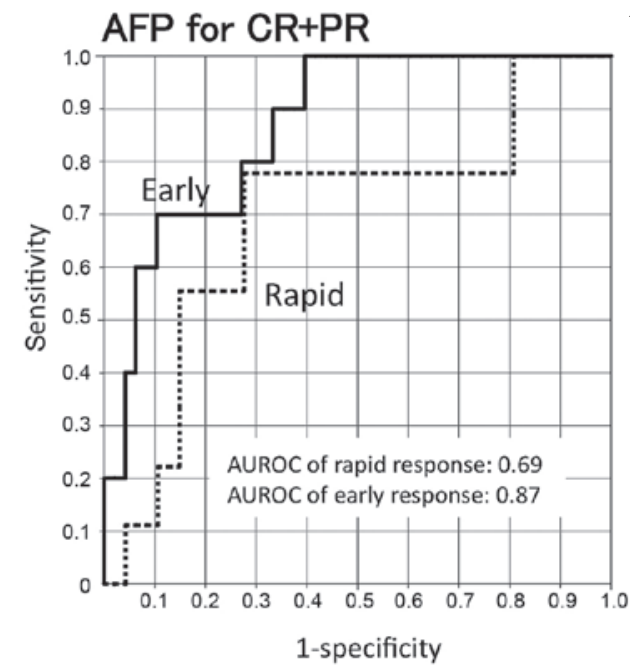

B DCP for CR

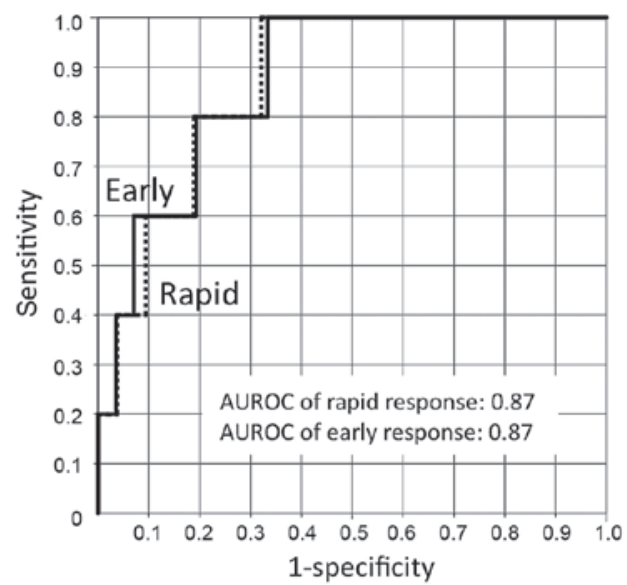

D

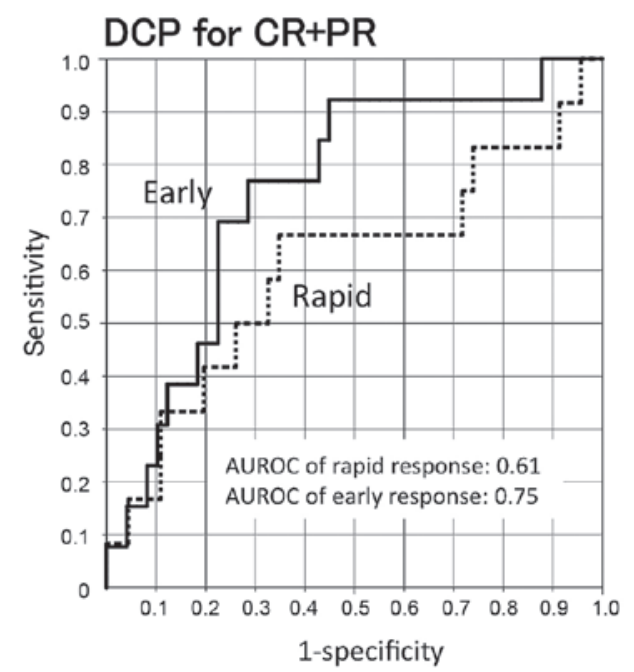

Figure 3. ROC curves of rapid and early response indices of AFP and DCP. Areas under ROC curves for early AFP or DCP responses were larger than those of rapid responses in the CR or CR+PR group (A-D), but were not significant. AFP, $\alpha$-fetoprotein; DCP, des- $\gamma$-carboxy prothrombin; AUROC, area under receiver operating curve; PR, partial response; $\mathrm{CR}$, complete response. 
Table IV. Predicting performance by combination of AFP and DCP.

\begin{tabular}{|c|c|c|c|c|c|}
\hline Characteristics & Sensitivity & Specificity & PPV & NPV & Hazard ratio \\
\hline \multicolumn{6}{|c|}{ Rapid AFP+DCP } \\
\hline $\mathrm{CR}$ & 0.67 & 0.98 & 0.67 & 0.98 & 88.0 \\
\hline $\mathrm{CR}+\mathrm{PR}$ & 0.63 & 0.88 & 0.50 & 0.92 & 11.6 \\
\hline \multicolumn{6}{|l|}{ Early AFP+DCP } \\
\hline $\mathrm{CR}$ & 1.00 & 1.00 & 1.00 & 1.00 & NA \\
\hline $\mathrm{CR}+\mathrm{PR}$ & 0.75 & 0.88 & 0.55 & 0.95 & 21.6 \\
\hline
\end{tabular}

PPV, positive predictive value; NPV, negative predictive value; AFP, $\alpha$-fetoprotein; DCP, des- $\gamma$-carboxy prothrombin; NA, not applicable; PR, partial response; $\mathrm{CR}$, complete response.

A

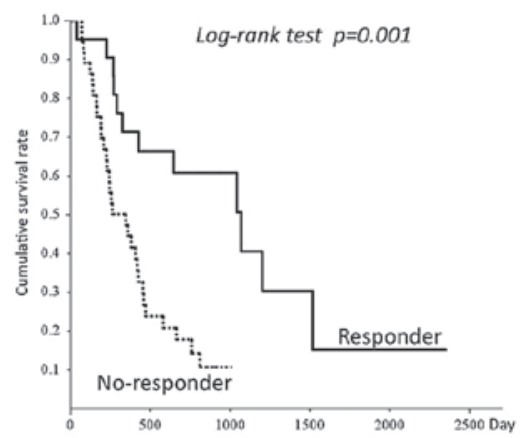

B

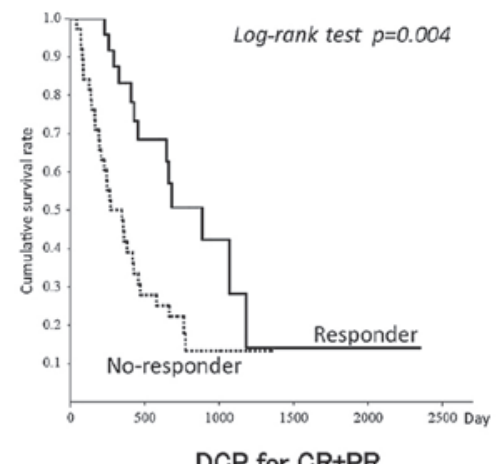

C

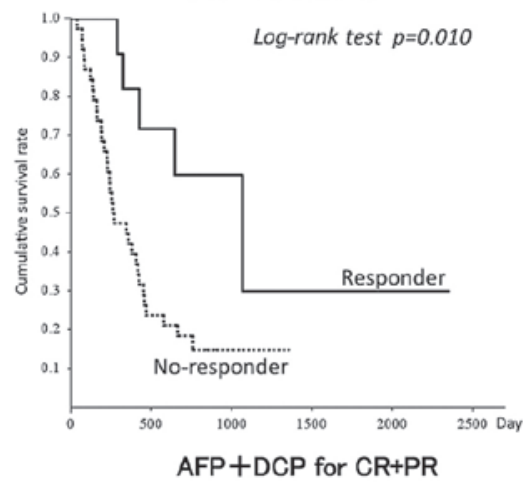

Figure 4. Kaplan-Meier analyses of overall survival according to AFP and DCP responses. (A) Early responders of AFP, (B) DCP and (C) AFP+DCP as predicting $\mathrm{CR}+\mathrm{PR}$ survived significantly longer compared to non-responders. AFP, $\alpha$-fetoprotein; DCP, des- $\gamma$-carboxy prothrombin; PR, partial response; $\mathrm{CR}$, complete response.

TAI + others, others and no treatment following initial TAI, respectively. All the patients without subsequent treatment had PD. Survival outcome in patients without subsequent treatment
Table V. Survival outcomes according to AFP and DCP responses.

\begin{tabular}{lll}
\hline & $\begin{array}{c}\text { Median } \\
\text { survival }\end{array}$ & \\
Characteristics & time, days & P-value \\
\hline AFP & & \\
Rapid & & \\
CR responder/non responder & $345 / 405$ & 0.146 \\
CR+PR responder/non responder & $380 / 291$ & 0.667 \\
Early & & \\
CR responder/non responder & $359 / 1,069$ & 0.015 \\
CR+PR responder/non responder & $345 / 1,069$ & 0.001 \\
DCP & & \\
Rapid & & \\
CR responder/non responder & $359 / 453$ & 0.402 \\
CR+PR responder/non responder & $269 / 456$ & 0.150 \\
Early & & \\
CR responder/non responder & $380 / 884$ & 0.056 \\
CR+PR responder/non responder & $269 / 884$ & 0.004 \\
AFP+DCP & & 0.134 \\
Rapid & & 0.062 \\
CR responder/non responder & $345 />2,000$ & 0.13 \\
CR+PR responder/non responder & $324 / 646$ & 0.134 \\
Early & & \\
CR responder/non responder & $345 / 1,069$ & 0.069 \\
CR+PR responder/non responder & $262 / 1,069$ & 0.06 \\
\hline
\end{tabular}

AFP, $\alpha$-fetoprotein; DCP, des- $\gamma$-carboxy prothrombin; NA, not applicable; $\mathrm{PR}$, partial response; $\mathrm{CR}$, complete response.

was significantly low compared to other treatments (data not shown, $\mathrm{P}<0.01$ ). There were no significant differences between repeated TAI, repeated TAI + others and others.

Survival outcomes according to AFP and DCP responses. Based on the cut-off values of $\mathrm{CR}$ and $\mathrm{CR}+\mathrm{PR}$, patients were divided into responder and non-responder groups to evaluate the prediction of survival outcome (Fig. 4, Table V). Early 
responders of $\mathrm{AFP}$ and $\mathrm{AFP}+\mathrm{DCP}$ for predicting $\mathrm{CR}$ survived significantly longer than non-responders $(\mathrm{P}<0.05)$. Early responders of $\mathrm{AFP}, \mathrm{DCP}$ and $\mathrm{AFP}+\mathrm{DCP}$ in predicting $\mathrm{CR}+\mathrm{PR}$ survived significantly longer than non-responders $(\mathrm{P}<0.05)$. Rapid responders of AFP, DCP and AFP+DCP were not significantly different from non-responders with regards to survival.

\section{Discussion}

Patients with advanced HCC who had early AFP and DCP responses following initial TAI had significantly improved treatment effects and longer overall survival than those without AFP and DCP responses.

AFP is secreted in $70 \%$ of patients with $\mathrm{HCC}$ and it is frequently measured in clinical practice for diagnosis or pretreatment prognosis, but the predictive or prognostic significance of the AFP response during treatment has not been evaluated frequently. DCP was also used as a tumor marker of HCC and was more likely to be elevated in patients with advanced HCCs, such as vascular invasion or distant metastasis (20). Investigators have proposed a role for tumor marker dynamics during surgical treatment, more specifically, that a change in AFP or DCP can predict prognosis or recurrence following surgical resection (21-23). However, the AFP and/or DCP responses have not been assessed in patients treated with TAI.

Three recent studies of patients with advanced HCC who received various types of systemic therapy, such as thalidomide (24), doxorubicin-based combination chemotherapy (15) and chemotherapy with or without molecularly targeted therapies (14), concluded that a change in AFP during treatment is a useful surrogate for predicting treatment effects and the survival rate of patients. The criteria for an AFP response have various definitions according to treatment modalities that affect AFP responses in different ways. Riaz et al (16) and Vora et al (14) defined an AFP response as $>50 \%$ reduction from baseline for locoregional therapy and systemic chemotherapy, whereas Chan et al (15) defined it as $>20 \%$ reduction in systemic chemotherapy. The present study identified that the rapid AFP and DCP responses in CR were 31 and $51 \%$ decreased from baseline, respectively. Early AFP and DCP responses in the same group were 80 and $88 \%$, respectively. These data were appropriate considering that the half-lives of AFP and DCP are 6 and 3.2 days, respectively (25).

Serum AFP and DCP levels have been routinely measured in the clinical setting for decades and they can be immediately, easily and broadly monitored in outpatient clinics as a potential surrogate for the effects of treatment in patients with advanced HCC. The present study found that the early AFP response was most sensitive for predicting $\mathrm{CR}$ and the sensitivity of rapid DCP for this group was $80 \%$. Early responders of AFP, DCP and a combination of these markers were shown to have improved survival outcomes than non-responders. To the best of our knowledge, this is the earliest time point used in similar studies and it was apparently the most relevant to the clinical treatment of advanced HCC. Treatment effects may be more accurately predicted at 1 month after the initial TAI as the areas under the ROC curves for AFP or DCP were larger for early, compared to the rapid responses.

The combination of AFP and DCP responses markedly increased the ability to predict $\mathrm{CR}$ and $\mathrm{CR}+\mathrm{PR}$ compared to each marker alone. One explanation may be that 2 of the 5 patients who achieved CR and 5 of 10 who achieved PR had high baseline values of either AFP or DCP.

A biomarker that can be detected during the early phase of treatment is valuable, as it may help to identify a subgroup of patients with a poor prognosis who would thus be candidates for other treatment strategies. Subsequent treatment methods following initial TAI except for no treatment did not affect treatment outcomes in terms of overall survival rate in the present study. Indeed, the predicting performance for all the treatments was similar to that for subsequent repeated TAI. At the advanced stage of HCC, multidisciplinary and personalized therapy was required. From this view point, predicting treatment outcomes in the early period following initial TAI is extremely important. The present results may aid physicians to accurately determine disease courses and establish further treatment plans following completion of the initial TAI. Thus, adding other types of systemic therapy, such as sorafenib, should be considered if possible at an earlier stage of treatment for patients with little or no AFP and DCP responses.

Several studies have examined the predictive roles of AFP and DCP responses (26-28), but the present findings are important for several reasons. First, to the best of our knowledge, only a few studies have simultaneously evaluated the clinical value of AFP and DCP responses through correlations with clinical outcomes in patients who were treated with homogeneous TAI protocols. Second, tumor markers were evaluated at an earlier time point than similar studies.

The potential limitations of the present study included a relatively small and less homogeneous patient population. AFP and DCP levels were measured only at baseline and at 1 and 4 weeks following TAI. Validation study in larger patient cohorts and/or with more stringent criteria for early AFP and DCP responses may help to verify the usefulness of such an approach.

In conclusion, early AFP and DCP responses appear to be significant predictors of the effects of TAI and the survival of patients with advanced HCC. Levels of AFP and DCP, particularly during the early phase of treatment, should be further explored in larger clinical studies.

\section{References}

1. El-Serag HB: Epidemiology of viral hepatitis and hepatocellular carcinoma. Gastroenterology 142: 1264-1273, 2012.

2. Ferlay J, Shin HR, Bray F, Forman D, Mathers C and Parkin DM: Estimates of worldwide burden of cancer in 2008: GLOBOCAN 2008. Int J Cancer 127: 2893-2917, 2010.

3. Blum HE: Hepatocellular carcinoma: Therapy and prevention. World J Gastroenterol 11: 7391-7400, 2005.

4. Furuse J: Sorafenib for the treatment of unresectable hepatocellular carcinoma. Biologics 2: 779-788, 2008.

5. Cheng AL, Kang YK, Chen Z, Tsao CJ, Qin S, Kim JS, Luo R, Feng J, Ye S, Yang TS, et al: Efficacy and safety of sorafenib in patients in the Asia-Pacific region with advanced hepatocellular carcinoma: A phase III randomised, double-blind, placebo-controlled trial. Lancet Oncol 10: 25-34, 2009.

6. Llovet JM, Ricci S, Mazzaferro V, Hilgard P, Gane E, Blanc JF, de Oliveira AC, Santoro A, Raoul JL, Forner A, et al; SHARP Investigators Study Group: Sorafenib in advanced hepatocellular carcinoma. N Engl J Med 359: 378-390, 2008.

7. Wörns MA, Weinmann A, Pfingst K, Schulte-Sasse C, Messow CM, Schulze-Bergkamen H, Teufel A, Schuchmann M, Kanzler S, Düber C, et al: Safety and efficacy of sorafenib in patients with advanced hepatocellular carcinoma in consideration of concomitant stage of liver cirrhosis. J Clin Gastroenterol 43: 489-495, 2009. 
8. Keating GM and Santoro A: Sorafenib: A review of its use in advanced hepatocellular carcinoma. Drugs 69: 223-240, 2009.

9. Ma YT and Palmer DH: Impact of restricting access to high-cost medications for hepatocellular carcinoma. Expert Rev Pharmacoecon Outcomes Res 12: 465-473, 2012.

10. Miyaki D, Aikata H, Honda Y, Naeshiro N, Nakahara T, Tanaka M, Nagaoki Y, Kawaoka T, Takaki S, Waki K, et al: Hepatic arterial infusion chemotherapy for advanced hepatocellular carcinoma according to Child-Pugh classification. J Gastroenterol Hepatol 27: 1850-1857, 2012.

11. Nouso K, Miyahara K, Uchida D, Kuwaki K, Izumi N, Omata M, Ichida T, Kudo M, Ku Y, Kokudo N, et al; Liver Cancer Study Group of Japan: Effect of hepatic arterial infusion chemotherapy of 5-fluorouracil and cisplatin for advanced hepatocellula carcinoma in the Nationwide Survey of Primary Liver Cancer in Japan. Br J Cancer 109: 1904-1907, 2013.

12. Bellet DH, Wands JR, Isselbacher KJ and Bohuon C: Serum alpha-fetoprotein levels in human disease: Perspective from a highly specific monoclonal radioimmunoassay. Proc Natl Acad Sci USA 81: 3869-3873, 1984.

13. Weitz IC and Liebman HA: Des-gamma-carboxy (abnormal) prothrombin and hepatocellular carcinoma: A critical review. Hepatology 18: 990-997, 1993.

14. Vora SR, Zheng H, Stadler ZK, Fuchs CS and Zhu AX: Serum alpha-fetoprotein response as a surrogate for clinical outcome in patients receiving systemic therapy for advanced hepatocellular carcinoma. Oncologist 14: 717-725, 2009.

15. Chan SL, Mo FK, Johnson PJ, Hui EP, Ma BB, Ho WM, Lam KC, Chan AT, Mok TS and Yeo W: New utility of an old marker: Serial alpha-fetoprotein measurement in predicting radiologic response and survival of patients with hepatocellular carcinoma undergoing systemic chemotherapy. J Clin Oncol 27: 446-452, 2009.

16. Riaz A, Ryu RK, Kulik LM, Mulcahy MF, Lewandowski RJ, Minocha J, Ibrahim SM, Sato KT, Baker T, Miller FH, et al: Alpha-fetoprotein response after locoregional therapy for hepatocellular carcinoma: Oncologic marker of radiologic response, progression, and survival. J Clin Oncol 27: 5734-5742, 2009

17. Yamasaki T, Kimura T, Kurokawa F, Aoyama K, Ishikawa T, Tajima K, Yokoyama Y, Takami T, Omori K, Kawaguchi K, et al: Prognostic factors in patients with advanced hepatocellular carcinoma receiving hepatic arterial infusion chemotherapy. J Gastroenterol 40: 70-78, 2005.

18. Yamamoto K, Imamura H, Matsuyama $Y$, Kume Y, Ikeda $H$, Norman GL, Shums Z, Aoki T, Hasegawa K, Beck Y, et al: AFP, AFP-L3, DCP and GP73 as markers for monitoring treatment response and recurrence and as surrogate markers of clinicopathological variables of HCC. J Gastroenterol 45: 1272-1282, 2010.

19. Kudo M, Kubo S, Takayasu K, Sakamoto M, Tanaka M, Ikai I, Furuse J, Nakamura K and Makuuchi M; Liver Cancer Study Group of Japan (Committee for Response Evaluation Criteria in Cancer of the Liver, Liver Cancer Study Group of Japan): Response Evaluation Criteria in Cancer of the Liver (RECICL) proposed by the Liver Cancer Study Group of Japan (2009 Revised Version). Hepatol Res 40: 686-692, 2010.
20. Tateishi R, Yoshida H, Matsuyama Y, Mine N, Kondo Y and Omata M: Diagnostic accuracy of tumor markers for hepatocellular carcinoma: A systematic review. Hepatol Int 2: 17-30, 2008.

21. Kim Y, Paik YH, Ahn SH, Youn YJ, Choi JW, Kim JK, Lee KS, Chon CY and Han KH: PIVKA-II is a useful tumor marker for recurrent hepatocellular carcinoma after surgical resection. Oncology 72 (Suppl 1): 52-57, 2007.

22. Yamamoto K, Imamura H, Matsuyama Y, Hasegawa K, Beck Y, Sugawara Y, Makuuchi M and Kokudo N: Significance of alpha-fetoprotein and des-gamma-carboxy prothrombin in patients with hepatocellular carcinoma undergoing hepatectomy. Ann Surg Oncol 16: 2795-2804, 2009.

23. Toyoda H, Kumada T, Kaneoka Y, Osaki Y, Kimura T, Arimoto A, Oka H, Yamazaki O, Manabe T, Urano F, et al: Prognostic value of pretreatment levels of tumor markers for hepatocellular carcinoma on survival after curative treatment of patients with HCC. J Hepatol 49: 223-232, 2008

24. Chen LT, Liu TW, Chao Y, Shiah HS, Chang JY, Juang SH, Chen SC, Chuang TR, Chin YH and Whang-Peng J: Alpha-fetoprotein response predicts survival benefits of thalidomide in advanced hepatocellular carcinoma. Aliment Pharmacol Ther 22: 217-226, 2005.

25. Kishi K, Sonomura T, Mitsuzane K, Nishida N, Kimura M, Satoh M, Yamada R, Kodama N, Kinoshita M, Tanaka H, et al: Time courses of PIVKA-II and AFP levels after hepatic artery embolization and hepatic artery infusion against hepatocellular carcinoma: Relation between the time course and tumor necrosis. Radiat Med 10: 189-195, 1992.

26. Lee MH, Kim SU, Kim Y, Ahn SH, Choi EH, Lee KH, Lee Y, Seong J, Han KH, Chon CY, et al: Early on-treatment predictions of clinical outcomes using alpha-fetoprotein and des-gamma-carboxy prothrombin responses in patients with advanced hepatocellular carcinoma. J Gastroenterol Hepatol 27: 313-322, 2012

27. Kim BK, Ahn SH, Seong JS, Park JY, Kim Y, Kim JK, Lee Y, Lee KH and Han KH: Early $\alpha$-fetoprotein response as a predictor for clinical outcome after localized concurrent chemoradiotherapy for advanced hepatocellular carcinoma. Liver Int 31: 369-376, 2011.

28. Shao YY, Lin ZZ, Hsu C, Shen YC, Hsu CH and Cheng AL: Early alpha-fetoprotein response predicts treatment efficacy of antiangiogenic systemic therapy in patients with advanced hepatocellular carcinoma. Cancer 116: 4590-4596, 2010. 